\title{
Effective charge-spin models for quantum dots
}

\author{
John H. Jefferson ${ }^{1}$ and Wolfgang Häusler ${ }^{2}$ \\ ${ }^{1}$ DRA, Electronics Sector, St. Andrews Road, Malvern, Worcs. WR14 3PS. U.K. \\ ${ }^{2}$ I. Institut für Theoretische Physik, Jungiusstr. 9, 20355 Hamburg, F. R. G.
}

(November 12, 2018)

\begin{abstract}
It is shown that at low densities, quantum dots with few electrons may be mapped onto effective charge-spin models for the low-energy eigenstates. This is justified by defining a lattice model based on a many-electron pocketstate basis in which electrons are localised near their classical ground-state positions. The equivalence to a single-band Hubbard model is then established leading to a charge-spin $(t-J-V)$ model which for most geometries reduces to a spin (Heisenberg) model. The method is refined to include processes which involve cyclic rotations of a "ring" of neighboring electrons. This is achieved by introducing intermediate lattice points and the importance of ring processes relative to pair-exchange processes is investigated using highorder degenerate perturbation theory and the WKB approximation. The energy spectra are computed from the effective models for specific cases and compared with exact results and other approximation methods.
\end{abstract}

71.10.-w , 71.23.An , 71.24.+q, 73.23.Hk

Typeset using REVTEX 


\section{INTRODUCTION}

Technological advances in microfabrication with corresponding reduction in feature sizes has led to renewed interest in the transport properties of semiconductor submicron structures both from the fundamental physics point of view and for possible future applications. In small metallic islands the Coulomb blockade gives rise to the single-electron effects, which may be modelled classically by a small intra-dot capacitance [1]. Islands fabricated on the basis of semiconductors, called quantum dots, show in addition to the charging effects discrete energy levels related to size quantisation [2 [4]. Individual quantum dots may be fabricated from hetero-structures, which limits the electron motion to two dimensions, by imposing lateral confinement with a metallic gate electrode deposited using fine-line (e-beam) lithography [4]. The discrete energy spectra may be measured in transport experiments at finite voltages [5, 6] or frequencies [7]. The influence of Coulomb correlations on the excitation energies is difficult to see optically by far infrared absorption [8] due to the generalized version of Kohn's theorem [9]. The observation of the quadrupole transitions by using grating couplers has been suggested [10] as a possible optical means.

Quantum dots have been referred to as 'artificial atoms' [11] since the number of conducting electrons can be smaller than ten or twenty. At the low electron densities which are experimentally accessible, the calculation of excitation energies becomes a challenging theoretical problem. For real atoms, an independent-electron picture correctly describes the main physics and the Hartree Fock approximation yields reasonably accurate eigenstates which may be refined in a controlled way by perturbation theory. This is not generally the case for semiconducting quantum dots for which even an optimal Hartree Fock approximation is significantly in error [12] and can even give qualitatively incorrect results, such as the wrong spin-multiplet structure. The reason for this qualitatively different behaviour is that the electrons in a semiconducting quantum dot are highly correlated, due to the low effective density and the restriction of the electron motion to only two dimensions. Many body effects must be taken into account. This has been done by numerically exact diagonalizations for systems with a very few electrons $(N \leq 4)$ [13. In order to obtain reasonably accurate spectra for systems with more electrons, approximations must be made. For electrons in high magnetic fields, the low-energy states of maximal spin have been determined to good accuracy by making a harmonic expansion about classical minimum energy configurations for circular dots with parabolic confining potentials [14]. The resulting effective Hamiltonian is then diagonalised explicitly and electron antisymmetry is subsequently imposed to determine the allowed eigenstates. High accuracy is achieved by including a sufficient number of Landau levels in the basis set. A complementary technique which has achieved some measure of success at low electron densities, and is not restricted to high magnetic fields, is based on many-electron "pocket" states [15]. Low-energy spectra of systems with up to six electrons have been determined accurately [16] and the agreement with the exact numerical solutions $(N \leq 4)$ is good. Unfortunately the computational effort is likely to become prohibitive for systems with more than ten electrons and we are again faced with the problem of devising a reliable approximation method for such cases.

One possible route is suggested by the pocket-state analysis itself which exploits the permutation symmetry of the wavefunctions. We notice that in many cases the eigensolutions may be represented by an effective spin model of a simple form (Heisenberg model). This 
apparent equivalence is reminiscent of magnetic insulators for which the correlated electron problem is known to reduce to a spin-Hamiltonian, a mapping which may be justified by transformation theory [17] or degenerate perturbation theory [18] as well as, in some cases, by the theory of permutation groups [19]. The magnetic insulator problem starts with a lattice model in which the electrons are localised on atomic-like orbitals and for which electron correlations are essential, the generic model being the so-called Hubbard model [20]. The underlying crystalline lattice establishes sites on which suitable one-electron states are centred. The question arises as to what circumstances, if any, might a Hubbard-type model be applicable to the problem of interacting electrons in a quantum dot, for which the Fermi wave lengths are much larger than the interatomic spacings and the underlying crystalline lattice loses its significance. At extremely low electron densities the long range interaction energy, according to Wigner [21], creates a crystalline like ground state which might define new electronic lattice sites. This crystallization is expected to take place only at electron densities which are a factor of 20 smaller than the densities used in experiments. Nevertheless, it has been shown that the assumption of localised electrons allows the calculation of discrete low energy excitations as quantum corrections to the Wigner crystal energies [16]. The validity of a Hubbard model description is not only an interesting question of principle but has the practical potential of enabling systems of many more electrons to be dealt with than has hitherto been the case, i.e. tens of electrons or more. This would enable the full machinery of techniques for solving the Hubbard and Heisenberg models to be applied, for which there has been much progress in recent years following the discovery of high-temperature superconductivity and the associated theoretical activity on correlated electron systems [22].

In this paper we will show that such a mapping may indeed be justified for the a priori continuous problem of interacting electrons in a quantum dot at low densities. This is done through the use of pocket-states which are briefly reviewed in the next section after introducing the basic interacting electron model. In section III we show how the pocketstates may be used to define a one-electron orthonormal basis with orbitals localised on a "lattice", related to the electron configuration(s) in the classical ground state (determined by electrostatics). This is then used to construct a tight-binding Hamiltonian which reduces to the Hubbard model at low electron densities. Further mapping to an effective (Heisenberg) spin Hamiltonian or charge-spin $(t-J-V)$ model is then performed.

In section IV we point out an essential difference between quantum dots and the corresponding correlated electron problem for interacting electrons on a true lattice of atoms, namely the increased importance of the so-called "ring" terms in the former case, which involve cyclic permutations of more than two electrons. The simple Hubbard model on a lattice underestimates the magnitude of these ring terms for the dot and it is shown how the method may be refined by introducing intermediate lattice points which are unoccupied in the ground manifold but give rise to the required ring processes through virtual excitations. In section $\mathrm{V}$ we solve the effective Hamiltonians for specific examples with $N \leq 6$ and compare with exact numerical and pocket-state results where appropriate. Finally, in section V1, we give a summary and discuss the outlook for this approach in dealing with systems of more electrons and of obtaining further corrections where necessary. 


\section{THE MODEL AND POCKET-STATE BASIS}

We consider the $N$-electron quantum dot described by the Hamiltonian

$$
\begin{gathered}
H=\sum_{i=1}^{N}\left(\frac{\boldsymbol{p}_{i}^{2}}{2 m}+v\left(\boldsymbol{x}_{i}\right)\right)+W\left(\boldsymbol{x}_{1} \ldots \boldsymbol{x}_{N}\right) \\
W\left(\boldsymbol{x}_{1} \ldots \boldsymbol{x}_{N}\right)=\frac{1}{2} \sum_{\substack{i, j \\
i \neq j}} w\left(\left|\boldsymbol{x}_{i}-\boldsymbol{x}_{j}\right|\right),
\end{gathered}
$$

where $\boldsymbol{x}_{i}$ and $\boldsymbol{p}_{i}$ are position and momentum of the $i$-th electron in $d$-dimensions $(d=2$ for most quantum dots) with (effective) mass $m$ and spin $s=1 / 2$. Neither the one-particle confinement potential $v(\boldsymbol{x})$ nor the interaction $w(x)$ depend explicitly on spin.

The cases of square well single particle potentials in one dimension (1D) and in two dimensions (for square geometry) have been discussed in [16. To be specific, and for comparison later, we shall also mainly confine ourselves to the $2 \mathrm{D}$ square potential well of square geometry, though the extension to other geometries is straightforward and, for the present consideration, the detailed form of $v(\boldsymbol{x})$ is not qualitatively important. At large mean inter-particle distances, $r_{\mathrm{s}}$, it becomes energetically favourable for the electron system to localize its charge density distribution in regions close to the classical ground-state electron configuration(s) [21,23], which may be determined by minimizing the electrostatic energy.

This fact is the motivation for the many-electron pocket-state basis in which the Hilbert space is restricted to $1 \leq p \leq \nu \cdot N$ ! basis states $|p\rangle$ with spatial representations being defined in configuration space of dimensionality $d N$. The number of classical minimum energy configurations $\nu$ may be greater than one in certain symmetric geometries. (For example, for $N=3$ on a square we have $\nu=4$ since, for the classical ground-state energy, any one of the four corners may be unoccupied.) The low-energy levels form a multiplet which contains $\nu \cdot 2^{N}$ states in total (including Zeeman levels). The excited 'vibrational' states scale like a power law $\sim r_{\mathrm{s}}^{-\gamma}$ with $r_{\mathrm{s}}(\gamma$ being close to $3 / 2)$ and can be neglected if the density is not too high. This is due to the scaling $\sim \exp \left(-\sqrt{r_{\mathrm{s}} / r_{\mathrm{c}}}\right)$ of the low energy excitations we are interested in here, where $r_{\mathrm{c}}$ is a length scale which characterizes the transition from the almost non-interacting situation $\left(r_{\mathrm{s}} \ll r_{\mathrm{c}}\right)$ into the qualitatively different regime of strong correlations $\left(r_{\mathrm{s}} \gg r_{\mathrm{c}}\right)$ [15].

In the pocket-state method, the first step is to calculate energies by assuming equivalent but distinguishable particles and ignoring their spin. Spin and statistics are subsequently reestablished by means of group theory. Tunneling integrals $\left\langle p|H| p^{\prime}\right\rangle$ between the localized many particle (pocket-) basis states determine the low-energy excitations. The tunneling processes correspond to (correlated) transitions between different particle arrangements. In the low-density regime one tunneling integral, $J / 2$, is exponentially larger than all others. In many cases the dominant $J$ corresponds to the exchange of only two particles, which may be adjacent in real space. Other processes could be the simultaneous (ring-) exchange of three or more particles, corresponding to a cyclic permutation.

An important feature of the spectra given by the pocket-state approximation (and by the effective charge-spin models to be derived and discussed in the following sections) is that 
the fine-structure of the energy spectra depends only on $J$, the magnitude of which may be estimated semiclassically within the multidimensional WKB approximation. The ratios between the energy differences are insensitive to the detailed form of the inter-electron potential and to $r_{\mathrm{s}}$. An examination of the spectra shows that these situations may be mapped onto an effective spin model with again one parameter for each process (pair exchange, ring) considered. This equivalence will be justified in the next section. Here we merely make the observation that for the cases considered by the pocket-state method, the energy spectrum is the same as that which would be given by a spin-Hamiltonian. An exception to this are situations for which more than one classical minimum energy configuration for the electrons exist $(\nu>1)$, such as are 2 or 3 electrons on a square. We will show in the next section that these latter situations may be mapped onto a so-called $t-J-V$ model in which the dominant tunneling process is now related to $t$, the amplitude corresponding to the jump of a single particle into an empty 'site'. $J$ is again an exchange process involving at least two particles and $V$ represents the Coulomb repulsion between electrons on neighboring 'sites'.

In general, the pocket-states are correlated electron states which need not be assumed as a direct product of one-electron states. However, we may mimic the eigenstates at sufficiently large $r_{\mathrm{s}}$ with a one-electron product basis within the Heitler London (HL) approximation. The latter are localised in regions close to the positions the electrons would have in the classical ground state. In the next section we show how this one-electron basis may be used to define an orthonormal basis from which we construct an effective Hubbard model. This effective Hamiltonian yields low-energy spectra which are at least as accurate as what would be given by the corresponding HL approximation using a product basis of non-orthogonal, one-electron wavefunctions. We then show in section IV how the method may be extended to cases where ring processes are important.

\section{MAPPING TO HUBBARD AND CHARGE-SPIN MODELS}

Let us assume that the pocket basis states $|p\rangle$ are approximated "optimally" by nonorthogonal one-electron wavefunctions $\phi_{i}$ centred at position $i$. These states have the form,

$$
\left\langle\boldsymbol{x}_{1}, \ldots, \boldsymbol{x}_{N} \mid p\right\rangle \equiv \phi_{p_{1}}\left(\boldsymbol{x}_{1}\right) \phi_{p_{2}}\left(\boldsymbol{x}_{2}\right) \ldots \phi_{p_{N}}\left(\boldsymbol{x}_{N}\right)
$$

where $\left\{p_{1}, \ldots, p_{N}\right\}$ is the permutation $p$ of the sequence $\{1, \ldots, N\}$. In this section we orthogonalise these one-electron wavefunctions and construct antisymmetrised $N$-electron states from which we define the effective Hubbard model, which is subsequently transformed into a spin-Hamiltonian. To illustrate the main features of the method with minimal mathematical complexity, we first consider in detail the simplest non-trivial case of two electrons in a 1D square well. The analysis is then generalised to $N$-electrons in one or two dimensions.

As is well known, for two electrons the antisymmetrised states may be written as the product of orbital and spin parts, as with the HL states of the hydrogen molecule. The symmetric (singlet) and antisymmetric (triplet) orbital states are thus,

$$
\Psi_{S}=\frac{\phi_{1}(1) \phi_{2}(2)+\phi_{2}(1) \phi_{1}(2)}{\sqrt{2\left(1+s^{2}\right)}}
$$


and

$$
\Psi_{A}=\frac{\phi_{1}(1) \phi_{2}(2)-\phi_{2}(1) \phi_{1}(2)}{\sqrt{2\left(1-s^{2}\right)}}
$$

where $s=\left\langle\phi_{1} \mid \phi_{2}\right\rangle$ is the overlap and the arguments $(i=1,2)$ abbreviate the coordinate $\boldsymbol{x}_{i}$ of the $i$-th particle. These HL states yield approximate singlet and triplet ground-state energies: $E_{\text {singlet }}=\left\langle\Psi_{S}|H| \Psi_{S}\right\rangle$ and $E_{\text {triplet }}=\left\langle\Psi_{A}|H| \Psi_{A}\right\rangle$, where $H$ is the Hamiltonian for two electrons given by equation (2.1).

We now transform to orthonormal one-electron states $\psi$ where

$$
\begin{gathered}
\psi_{1}=\left(\frac{1}{2 \sqrt{1+s}}+\frac{1}{2 \sqrt{1-s}}\right) \phi_{1}+\left(\frac{1}{2 \sqrt{1+s}}-\frac{1}{2 \sqrt{1-s}}\right) \phi_{2} \\
\psi_{2}=\left(\frac{1}{2 \sqrt{1+s}}+\frac{1}{2 \sqrt{1-s}}\right) \phi_{2}+\left(\frac{1}{2 \sqrt{1+s}}-\frac{1}{2 \sqrt{1-s}}\right) \phi_{1} .
\end{gathered}
$$

Inverting this transformation and substituting into equations (3.2) and (3.3) we get,

$$
\begin{aligned}
& \Psi_{S}=\frac{1}{\sqrt{1+s^{2}}} \Psi_{S}^{(1)}+\frac{s}{\sqrt{1+s^{2}}} \Psi_{S}^{(2)} \\
& \Psi_{S}^{(1)}=\frac{\psi_{1}(1) \psi_{2}(2)+\psi_{2}(1) \psi_{1}(2)}{\sqrt{2}} \\
& \Psi_{S}^{(2)}=\frac{\psi_{1}(1) \psi_{1}(2)+\psi_{2}(1) \psi_{2}(2)}{\sqrt{2}}
\end{aligned}
$$

and

$$
\Psi_{A}=\frac{\psi_{1}(1) \psi_{2}(2)-\psi_{2}(1) \psi_{1}(2)}{\sqrt{2}} .
$$

We note that $\Psi_{A}$ (triplet) has the same form as for the non-orthogonal states given in equation (3.3), i.e. it corresponds to one electron in each orbital. On the other hand $\Psi_{S}$ (singlet) consists of two components, one with an electron on each orbital $\left(\Psi_{S}^{(1)}\right)$ and the other with both electrons in the same orbital $\left(\Psi_{S}^{(2)}\right)$. (The latter state is not allowed for the triplet by the Pauli principle.) This "double occupation" is thus a direct consequence of orthogonalisation.

Using these two-electron states composed of orthogonal one-electron wavefunctions will, of course, yield exactly the same approximate singlet and triplet energy expectation values. However, we can obtain a more accurate estimate for the ground-state energy of the singlet by diagonalising the Hamiltonian in the Hilbert space defined by the two-electron base states $\Psi_{S}^{(1)}$ and $\Psi_{S}^{(2)}$ (Eqn. 3.7). This follows from the variational principle which ensures that diagonalisation of the Hamiltonian matrix will yield the optimum superposition of the base states, whereas the HL state (3.6) is not optimum in general. 
This principle also applies to the general $N$-electron case as stated in the introduction, i.e. diagonalisation of the Hamiltonian in the restricted Hilbert space defined by base states (Slater determinants) constructed from orthogonalised one-electron wavefunctions will yield a more accurate low-energy spectrum than the simple products of non-orthogonal one-electron states (3.2) and (3.3).

For the two-electron case the $(2 \times 2)$ Hamiltonian singlet matrix is,

$$
H_{\mathrm{s}}=\left(\begin{array}{cc}
2 \varepsilon+\underset{\tilde{t}^{*}}{V+j} & 2 \tilde{t} \\
2 \varepsilon+U+\tilde{\jmath}
\end{array}\right)
$$

where

$$
\begin{aligned}
\varepsilon & =\left\langle\psi_{1}\left|\left[\frac{\boldsymbol{p}^{2}}{2 m}+v\right]\right| \psi_{1}\right\rangle=\left\langle\psi_{2}\left|\left[\frac{\boldsymbol{p}^{2}}{2 m}+v\right]\right| \psi_{2}\right\rangle \\
V & =\int\left|\psi_{1}(\boldsymbol{x})\right|^{2}\left|\psi_{2}(\boldsymbol{y})\right|^{2} w(|\boldsymbol{x}-\boldsymbol{y}|) \mathrm{d}^{3} x \mathrm{~d}^{3} y \\
U & =\int\left|\psi_{1}(\boldsymbol{x})\right|^{2}\left|\psi_{1}(\boldsymbol{y})\right|^{2} w(|\boldsymbol{x}-\boldsymbol{y}|) \mathrm{d}^{3} x \mathrm{~d}^{3} y=\int\left|\psi_{2}(\boldsymbol{x})\right|^{2}\left|\psi_{2}(\boldsymbol{y})\right|^{2} w(|\boldsymbol{x}-\boldsymbol{y}|) \mathrm{d}^{3} x \mathrm{~d}^{3} y, \\
t & =\int \psi_{1}^{*}(\boldsymbol{x})\left[\frac{\boldsymbol{p}^{2}}{2 m}+v(\boldsymbol{x})\right] \psi_{2}(\boldsymbol{x}) \mathrm{d}^{3} x \\
\tau & =\int \psi_{1}^{*}(\boldsymbol{x})\left[\int\left|\psi_{1}(\boldsymbol{y})\right|^{2} w(|\boldsymbol{x}-\boldsymbol{y}|) d^{3} y\right] \psi_{2}(\boldsymbol{x}) \mathrm{d}^{3} x \\
\tilde{t} & =t+\tau, \\
j & =\int \psi_{1}^{*}(\boldsymbol{x}) \psi_{2}^{*}(\boldsymbol{y}) w(|\boldsymbol{x}-\boldsymbol{y}|) \psi_{2}(\boldsymbol{x}) \psi_{1}(\boldsymbol{y}) \mathrm{d}^{3} x \mathrm{~d}^{3} y \\
\xi & =\int \psi_{2}^{*}(\boldsymbol{x}) \psi_{2}^{*}(\boldsymbol{y}) w(|\boldsymbol{x}-\boldsymbol{y}|) \psi_{1}(\boldsymbol{x}) \psi_{1}(\boldsymbol{y}) \mathrm{d}^{3} x \mathrm{~d}^{3} y \\
\tilde{\jmath} & =\operatorname{Re}(\xi) \quad .
\end{aligned}
$$

Note, $j=\xi=\tilde{\jmath}$ if the $\psi_{i}$ are real.

The triplet energy is,

$$
E_{t}=2 \varepsilon+V .
$$

Within this restricted manifold of states, the singlet-triplet Hamiltonian matrix, equations (3.10) and (3.20), are equivalent to an effective Hamiltonian,

$$
H_{\mathrm{eff}}=\varepsilon\left[n_{1}+n_{2}\right]+U\left[n_{1 \uparrow} n_{1 \downarrow}+n_{2 \uparrow} n_{2 \downarrow}\right]+V n_{1} n_{2}+j \sum_{\sigma} c_{1 \sigma}^{\dagger} c_{2 \bar{\sigma}}^{\dagger} c_{2 \sigma} c_{1 \bar{\sigma}}
$$




$$
+\left[\sum_{\sigma}\left(t c_{1 \sigma}^{\dagger} c_{2 \sigma}+\tau\left(c_{1 \sigma}^{\dagger} c_{2 \sigma} n_{1 \bar{\sigma}}+c_{2 \sigma}^{\dagger} c_{1 \sigma} n_{2 \bar{\sigma}}\right)+\frac{1}{2} \xi c_{1 \sigma}^{\dagger} c_{1 \bar{\sigma}}^{\dagger} c_{2 \bar{\sigma}} c_{2 \sigma}\right)+\mathrm{hc}\right]
$$

where $c_{i \sigma}^{\dagger}$ is a Fermi creation operator at 'site' $i=1,2$ satisfying $\left\langle\boldsymbol{x}\left|c_{i \sigma}^{\dagger}\right| \operatorname{vac}\right\rangle=\psi_{i \sigma}(\boldsymbol{x})$; $n_{i \sigma}=c_{i \sigma}^{\dagger} c_{i \sigma}$ and $n_{i}=n_{i \uparrow}+n_{i \downarrow}$.

This effective Hamiltonian is very similar to that considered by Hubbard [20] for a periodic array of one-electron atoms in the study of the metal-insulator (Mott) transition. Indeed, for this two-electron case, equation (3.21) is an effective Hamiltonian for the hydrogen molecule within a restricted Hilbert space of (effective) 1s-orbitals. We stress, however, that in the quantum dot case the localised orbitals are fundamentally different from those in the atomic case in that their very existence depends on the electron-electron repulsion which is responsible for localising the electrons near specific points in real space at low density. For this reason we are not justified, a priori, in dropping all but the largest Coulomb term $(U)$, as is usually done in the Hubbard model. In particular, the nearest-neighbor effective Coulomb interaction, $V$, becomes most important when $r_{\mathrm{s}}$ becomes very large and equation (3.21) simply reduces to the classical expression for the ground-state energy, independent of spin, as it should. This may be seen more clearly by transforming (3.21) into an effective spin-Hamiltonian which is demonstrated most directly for this two-electron case by diagonalising the singlet-matrix, (3.10), explicitly to yield eigenenergies:

$$
E_{ \pm}=\frac{1}{2}\left[4 \varepsilon+V+U+j+\tilde{\jmath} \pm \sqrt{(U-V+\tilde{\jmath}-j)^{2}+16|\tilde{t}|^{2}}\right] .
$$

Since for the cases of interest (large separation between the electrons and real one-particle wave functions), $U \gg V, j, \tilde{\jmath}$ and $j=\tilde{\jmath}$, then,

$$
E_{+} \approx 2 \varepsilon+U+j+\frac{4|\tilde{t}|^{2}}{U-V}
$$

and

$$
E_{-} \approx 2 \varepsilon+V+j-\frac{4|\tilde{t}|^{2}}{U-V}
$$

Combining these equations with (3.20) for the triplet we see that the energy spectrum consists of a singlet-triplet pair at low energies separated by a singlet at energy $\sim U$ higher. Hence the low-energy singlet-triplet is equivalent to a spin system with effective spin-Hamiltonian,

$$
H_{\text {spin }}=2 \varepsilon+V+J\left(s_{1} \cdot s_{2}-1 / 4\right)
$$

where

$$
J=\frac{4|\tilde{t}|^{2}}{U-V}-j \approx \frac{4|\tilde{t}|^{2}}{U}-j
$$

The first term in equation (3.26), favouring a singlet ground-state, is sometimes referred to as 'superexchange' and is usually larger than the second 'direct' exchange term, $j$. This 
superexchange contribution has its origins in the assumed non-orthogonality of the initial Heitler-London basis $\phi_{i}$ in (3.1). From the pocket states it is known that they form a non-orthogonal basis set for the low energy eigenstates [24].

We now consider the general case of $N$ electrons in a quantum dot. Starting with a suitable product-basis of pocket-states, equation (3.1), we may, in general, define an orthonormal basis by Löwdin's method [25]. (For high-symmetry situations it may be more convenient and expedient to use some other method. For example, in the case of four electrons on a square we have $\pi / 4$ rotational symmetry and we may generate orthonormal states by forming molecular orbitals, normalising and then transforming back to localised states. Similarly, for translationally invariant systems we may transform to running (Bloch) waves, normalise and then transform back to localised (Wannier) states.) Within the corresponding many-electron Hilbert space we may express the Hamiltonian in second quantised form. We are led directly to an effective 'single-band' model of the type considered by Hubbard, generalising equation (3.21),

$$
\begin{aligned}
H_{\mathrm{eff}}= & \sum_{i}\left[\varepsilon_{i} n_{i}+U_{i} n_{i \uparrow} n_{i \downarrow}\right]+\sum_{i j} V_{i j} n_{i} n_{j}+\sum_{i j \sigma} j_{i j} c_{i \sigma}^{\dagger} c_{j \bar{\sigma}}^{\dagger} c_{j \sigma} c_{i \bar{\sigma}} \\
& +\left[\sum_{i j \sigma}\left(t_{i j} c_{i \sigma}^{\dagger} c_{j \sigma}+\tau_{i j}\left(c_{i \sigma}^{\dagger} c_{j \sigma} n_{i \bar{\sigma}}+c_{j \sigma}^{\dagger} c_{i \sigma} n_{j \bar{\sigma}}\right)+\frac{1}{2} \xi_{i j} c_{i \sigma}^{\dagger} c_{i \bar{\sigma}}^{\dagger} c_{j \bar{\sigma}} c_{j \sigma}\right)+\mathrm{hc}\right]
\end{aligned}
$$

where the indices $i$ and $j$ should be distinct. The parameters $\varepsilon, U, V, j, t, \tau$ and $\xi$ have the same meaning as in the two-electron case (3.11), though it should be noted that they will, in general, depend on the position of the electron, or pair of electrons and on separation. In particular the parameters $j, t, \tau$ and $\xi$ related to electron transfers decay rapidly as the distance between the 'sites' $i$ and $j$ increases. This allows us to retain only nearest neighbor terms in the double summations. As with the case of two electrons, we may eliminate the high-energy states of $H_{\text {eff }}$ to yield an effective spin-Hamiltonian. This may be done using degenerate perturbation theory [26] or a canonical transformation [27], as with the case of a lattice.

Specifically in the situations of two (or higher) dimensional quantum dots the cases of more than one minimum of the classical electrostatic energy may occur $(\nu>1)$. Then the quantum mechanical charge density distribution shows more than $N$ peaks at large $r_{\mathrm{s}}$, say $\tilde{N}>N$. The number of single electron states must be equal to $\tilde{N}$ in these cases. $\tilde{N}-N$ lattice sites will be unoccupied at an instant. Consider, for example, the case of a square confining potential in two dimensions. For $N=2, \tilde{N}=4$ and the electrons will be found mainly near opposite vertices along the diagonal of the square, due to the Coulomb repulsion. States with two electrons near adjacent vertices will be of order $V$ higher in energy (nearest-neighbor Coulomb repulsion energy) and states with two electrons located near the same vertex will be of order $U$ higher in energy. Similarly for $N=3$ there will be low-lying states with electrons located near three of the $\tilde{N}=4$ vertices with states having two electrons near the same vertex again being $\sim U$ higher in energy. For $N=4$ all vertices are occupied in the ground manifold, with configurations for which there are two electrons near one vertex being at least $\sim U$ higher in energy.

In each of these cases the high-energy states, corresponding to one or more vertices being occupied by two electrons, may be eliminated from the Hilbert space by degenerate 
perturbation theory, where they appear as intermediate states. More precisely, we first write $H_{\text {eff }}$ in the form $H_{\text {eff }}=H_{0}+H_{1}$ where $H_{0}=\sum_{i}\left[\varepsilon_{i} n_{i}+U_{i} n_{i \uparrow} n_{i \downarrow}\right] . H_{0}$ is diagonal in the basis of product states with the highest-energy states having two electrons in the same state, being $\sim U$ higher than the low-energy states [28]. Eliminating these states to second-order results in an effective ' $t-J-V$ ' Hamiltonian [29],

$$
H_{\mathrm{eff}}^{t J V}=P \sum_{i j}\left[\sum_{\sigma}\left(t_{i j} c_{i \sigma}^{\dagger} c_{j \sigma}+\mathrm{hc}\right)+J_{i j}\left(\boldsymbol{s}_{i} \cdot \boldsymbol{s}_{j}-1 / 4\right) n_{i} n_{j}+V_{i j} n_{i} n_{j}\right] P
$$

In this equation $t$ and $V$ have the same meaning as before and

$$
J_{i j}=2\left|t_{i j}+\tau_{i j}\right|^{2}\left[\frac{1}{U_{i}}+\frac{1}{U_{j}}\right]-j_{i j} .
$$

$P$ is a projection operator which eliminates all base states for which there are two electrons in the same localised state. (These states appear as intermediate states and give rise to the 'superexchange' in equation (3.29).) We also note that the $\varepsilon, \tau$ and $\xi$ terms in equation (3.27) have disappeared in equation (3.28). The former has been dropped since it always gives rise to a constant and the latter two are precluded by $P$ (as is the $U$-term) since they would always give rise to double occupation at a 'site'.

Equations (3.27) and (3.28) are the fundamental effective Hamiltonians for interacting electrons at low-density in a quantum dot and capture the main physics of the problem for most situations. (They do not, however, include processes in which groups of electrons can rotate simultaneously, which can be important in some circumstances. The generalisation to include these so-called ring terms is given in the next section.) Although these effective models were justified by starting with the Heitler-London approximation to pocket states using non-orthogonal one-electron states, we emphasize that they have a greater range of validity than the initial approximation suggests. The above methodology enabled us to define a basis set of product states (Slater determinants) of some one-electron states and this led directly to effective Hamiltonians operating within this restricted (incomplete) basis set. However, it is well known [26], that higher-lying base states may be accounted for by perturbation theory. This technique has recently been applied very successfully to lattice models in which an effective single-band Hubbard model was derived from a multi-band model using quasi-degenerate perturbation theory [30]. We shall not attempt such a reduction here for the quantum dot problem, but merely point out that its main effect would be to renormalise the effective parameters $\left(\varepsilon_{i}, U_{i}, t_{i j}\right.$ etc) and extend the validity of the resulting models.

Let us apply (3.28) to the case of electrons in a square well in two dimensions, discussed above. For $N=2$ and $N=3$ the $t$-term will cause electrons to hop from occupied to unoccupied sites whereas the $J$ and $V$ terms will be effective only when two adjacent sites are occupied. The presence of the $V$-term ensures that for $N=2$ the electrons will have lower energy when they are located on diagonally opposite vertices of the well, as mentioned earlier. For $N=4$ the $t$-term may be omitted since all four vertices are occupied in the ground manifold and it would thus give rise to double occupancy, precluded by $P$. The $V$-term may also be dropped since it gives rise to a constant contribution. Hence, the sites $i$ and $j$ can be restricted to nearest neighbors so that (3.28) reduces to a Heisenberg model, 


$$
H_{J}=\sum_{<i j>} J_{i j} \boldsymbol{s}_{i} \cdot \boldsymbol{s}_{j}
$$

where $P$ has been omitted for brevity.

Similar considerations apply for $N>4$ and the effective Hamiltonian will either have the form (3.30) or (3.28), depending on whether $\nu=1$ (e.g. $N=4,5)$ or $\nu>1$ (e.g. $N=2,3,6)$. For the latter cases a further reduction will be possible when some of the states of the $t-J-V$ model are clearly higher in energy due to the $V$-interaction.

Consider, for example, the case two electrons in a square-shaped dot for which there are six ways of distributing the two (indistinguishable) electrons in the four corners, resulting in a Hilbert space of dimension $4 \times 6=24$ when spin is taken into account. Four of these configurations (16 with spin) are of order $V$ higher in energy than the remaining two, as shown in figure 1. These high-energy base states may be eliminated from the problem by a further application of degenerate perturbation theory, resulting in an effective Hamiltonian which only operates in the Hilbert space of the 8 lowest-energy base states. By a straightforward calculation this takes the form (apart from an unimportant overall constant):

$$
H_{\text {eff }}=\Delta\left(n_{1} n_{3}-n_{2} n_{4}\right)\left(R_{\pi / 2}-R_{-\pi / 2}\right)+J\left[\left(\mathbf{s}_{1} \cdot \mathbf{s}_{3}-1 / 4\right) n_{1} n_{3}+\left(\mathbf{s}_{2} \cdot \mathbf{s}_{4}-1 / 4\right) n_{2} n_{4}\right]
$$

where $\Delta=2 t^{2} / V$ and $R_{\theta}$ is a rotation operator, i.e.

$$
R_{\pi / 2}\left|\sigma, *, \sigma^{\prime}, *\right\rangle=\left|*, \sigma, *, \sigma^{\prime}\right\rangle \quad,
$$

etc. Note that the prefactor $\left(n_{1} n_{3}-n_{2} n_{4}\right)$ takes the value \pm 1 depending on whether either 'sites' 1 and 3, or 2 and 4 are occupied. This effective Hamiltonian is easily diagonalised. The spectrum consists of two singlets and two triplets. We see immediately that (3.31) gives zero when operating on a spin-polarised state and hence the triplets are at zero energy with the singlets at energies $-J \pm 2 \Delta$, in agreement with what is obtained from the pocket state approximation for this problem when the exchange of the two electrons is included there [16]. There are thus two contributions to the 'binding energy' of the singlet ground state, a resonance energy $(-2 \Delta)$ and a superexchange energy $(-J)$. It is easy to see that the former will be dominant since it occurs in second-order and does not involve doubleoccupation of a 'site'. On the other hand, there are two contributions to $J$, the second-order term $\sim 4 t_{13}^{2} / U$ and fourth-order terms $\sim t_{12}^{4} / V^{2} U$. We may extend these considerations to a regular polygon with $N$ vertices and $N / 2$ electrons. The effective Hamiltonian will again consist of rotation operators (of $\pm 2 \pi / N$ ) and exchange terms. The exchange terms are independent of $N$, whereas the rotation terms becomes relatively less important since they first occur in $N$ th-order. In the next section we shall encounter circumstances where simultaneous rotations of more than two electrons may be important.

We conclude this section by emphasizing the computational advantage in this reduction to a spin or charge-spin Hamiltonian. Thus, for example, the Heisenberg model lies in a Hilbert space of dimension $2^{N}$ for $N$ electrons, which may be further reduced by exploiting other symmetries, e.g. conservation of total $S_{z}$ which reduces the largest subspace to $N ! /\left(\left(\frac{N}{2}\right) !\right)^{2}\left(S_{z}=0\right.$ for $N$ even). This should be compared with

$$
\max _{S}\left[\frac{(2 S+1) N !}{(N / 2+S+1) !(N / 2-S) !}\right]^{2}
$$


for the largest subspace of the Hamiltonian in the original pocket-state basis.

The mapping also gives interesting insight into the nature of the energy spectrum. For those cases which reduce to a Heisenberg model, the spectrum consists of spin-multiplets with energy-separation of order $J$ ('bandwidth' $\sim 2 N J)$. On the other hand, for cases which reduce to a charge-spin model, there are multiplets separated by energies of order $t \gg J$ in the same geometry and electron densities), each of which have a fine structure with energy splittings of order $J$.

The forgoing analysis for electrons may also be extended to a fictitious system of spin- $1 / 2$ charged bosons. For repulsive Coulomb interactions they also show Wigner crystallization and the pocket-state method can be applied. This problem was considered by Häusler [16] in order to proof the state of highest spin within the multiplet structure of electrons (fermions) in a quantum dot being the spin polarized state for $\nu=1$ by exploiting an isomorphism between fermions and bosons using permutation group theory. We reconsider this problem here to enable another direct comparison with the pocket-state description.

The case of spin-1/2 bosons is similar to the fermion case in that at low densities the largest 'Coulomb' matrix elements correspond to two bosons on the same 'site'. Neglecting all other matrix elements thus gives the 'boson' Hubbard model:

$$
H=\sum_{<i j>\sigma}\left(t_{i j} b_{i \sigma}^{\dagger} b_{j \sigma}+H . c .\right)+\frac{U}{2} \sum_{i, \sigma \sigma^{\prime}} b_{i \sigma}^{\dagger} b_{i \sigma^{\prime}}^{\dagger} b_{i \sigma^{\prime}} b_{i \sigma}
$$

Using the usual commutation rules for the Bose-operators yields

$$
\begin{gathered}
b^{\dagger}|n\rangle=\sqrt{n+1}|n+1\rangle \\
b|n\rangle=\sqrt{n}|n-1\rangle .
\end{gathered}
$$

It follows that:

$$
h_{0}\left|\sigma, \sigma^{\prime}\right\rangle=U\left|\sigma, \sigma^{\prime}\right\rangle
$$

where

$$
h_{0}=\frac{U}{2} \sum_{\sigma \sigma^{\prime}} b_{\sigma}^{\dagger} b_{\sigma^{\prime}}^{\dagger} b_{\sigma^{\prime}} b_{\sigma}
$$

This is the same result as the case of fermions except that with bosons the two particles may have the same spin.

As with the fermion case, we can now reduce (3.33) to a Heisenberg or $t-J-V$ model (depending on whether or not the number of bosons equals the number of lattice sites). Let us, for simplicity, do this for just two sites. The extension to the general case may be done by perturbation theory in the same way as with fermions.

In the 'atomic limit' $(t=0)$ the eigenstates of (3.33) with two bosons consist of either one boson on each site with either spin (energy 0) or one site unoccupied and the other doubly occupied, again with either spin (energy $=U$ ). Using these base states it is straightforward to show that the singlet state:

$$
\left|\psi_{S}\right\rangle=\frac{|\uparrow, \downarrow\rangle-|\downarrow, \uparrow\rangle}{\sqrt{2}}
$$


is unaffected by the hopping, i.e.

$$
H\left|\psi_{S}\right\rangle=0
$$

On the other hand the triplet states mix. For example, using (3.34), we get:

$$
\begin{aligned}
& H|\uparrow, \uparrow\rangle=t[|\cdot, \uparrow \uparrow\rangle+|\uparrow \uparrow, \cdot\rangle], \\
& H|\uparrow \uparrow, \cdot\rangle=U|\uparrow \uparrow, \cdot\rangle+\sqrt{2} t|\uparrow, \uparrow\rangle
\end{aligned}
$$

etc. This leads directly to the triplet Hamiltonian matrix:

$$
\left[\begin{array}{ll}
0 & 2 t \\
2 t & U
\end{array}\right]
$$

with lowest eigenvalue:

$$
E=\frac{U-\sqrt{U^{2}+16 t^{2}}}{2}
$$

This is exactly the same result as for the fermions except that the positions of the (lowest) singlet and triplet levels are reversed. It follows that the lowest singlet and triplet is equivalent to a Heisenberg model with ferromagnetic exchange of the same magnitude as the case of fermions. As mentioned above, this may be generalised to any number of sites by degenerate perturbation theory resulting in a Heisenberg model in which $J \rightarrow-J$ when compared with the fermion case, leading to an 'inverted' spectrum in agreement with the permutation group analysis described in the second reference of [16].

In a similar fashion, cases for which the number of bosons is less than the number of sites (such as 2 or 3 on a square) may be reduced to a $t-J-V$ model. However, whilst the sign of $J$ is reversed relative to the case of fermions, the sign of $t$ remains the same and the resulting spectrum is no longer a simple inversion.

\section{RING TERMS}

In the section [II] it was shown that the $t-J-V$ model may be reduced to an effective Hamiltonian which only involves the lowest-energy base states. This effective Hamiltonian then contains operators which rotate some or all of the electrons simultaneously (cf. (3.31)). The possibility of such a collective motion of a subset or all of the electrons is much more general and will in fact occur for any geometry. These processes are negligible within the framework of the usual lattice models but this is not always the case in quantum dots or other semicondutor based nano-structures where such 'ring' terms can become dominant. As we shall see they may be included in a lattice model by introducing intermediate lattice points which are unoccupied in the ground manifold.

Consider, for example, the case of an equilateral triangular dot containing three electrons, as shown in figure 2. Using the Hilbert space defined by the Heitler-London approximation

to the pocket-states, leads directly to the Heisenberg model for this system, with a spin-1/2 
ground state, as shown in section [II]. Ring terms corresponding to cyclic rotations of all three electrons are not included in this model. However, it is clear from the pocket-state description that such processes exist. The relevant tunneling integrals between the $3 !=6$ pocket-states in this case can be represented by permutations of the types

$$
\begin{array}{llll}
(123) \longrightarrow(213) & : & J / 2 & \text { Pair Exchange } \\
(123) \longrightarrow(312) & : & K / 2 & \text { Ring Exchange } .
\end{array}
$$

Their magnitudes may be estimated semiclassically within the WKB approximation. The most important contributions to $J$ and $K$ vary exponentially with the particle distance $r_{\mathrm{s}}$ (which equals the length of one side of the triangle in the case considered), i.e.

$$
J / 2 \sim \mathrm{e}^{-\mathcal{S}_{(123) \rightarrow(213)}} \quad, \quad K / 2 \sim \mathrm{e}^{-\mathcal{S}_{(123) \rightarrow(312)}}
$$

where

$$
\mathcal{S}=\int_{0}^{1} \mathrm{~d} q \sqrt{2 m n W(\overrightarrow{\boldsymbol{x}}(q))}
$$

is the action associated with the transport of $n$ particles $(n=2$ or $n=3$ for $J$ or $K$, respectively) of mass $m$ from the initial classical ground state (123) into the final state. The transition takes place along the trajectory $\overrightarrow{\boldsymbol{x}}(q)$ which in principle obeys an equation of motion which minimises the classical action. Here, it is parametrized by $0 \leq q \leq 1$ in configuration space (of dimensionality $d N=6$ ). For an explicit estimate let us specify the interaction between the particles and assume $w\left(\left|\boldsymbol{x}_{i}-\boldsymbol{x}_{j}\right|\right)=\frac{e^{2} / \kappa}{\left|\boldsymbol{x}_{i}-\boldsymbol{x}_{j}\right|}$. Then $W(\overrightarrow{\boldsymbol{x}})$ is the Coulomb energy at $\overrightarrow{\boldsymbol{x}} \equiv\left\{\boldsymbol{x}_{1}, \boldsymbol{x}_{2}, \boldsymbol{x}_{3}\right\}$ where the equilibrium value, $3 \frac{e^{2} / \kappa}{r_{\mathrm{s}}}$, has been subtracted so that $W(\overrightarrow{\boldsymbol{x}}(0))=W(\overrightarrow{\boldsymbol{x}}(1))=0$. The results

$$
\begin{aligned}
\mathcal{S}_{(123) \rightarrow(213)} & =4 \sqrt{m \frac{e^{2}}{\kappa} r_{\mathrm{s}}} \int_{0}^{1 / 2} \mathrm{~d} q\left[\frac{1}{\sqrt{1-q+q^{2}}}+\frac{1}{\sqrt{1-\frac{29}{8} q+4 q^{2}}}+\frac{1}{\sqrt{1-\frac{5}{2} q+\frac{7}{4} q^{2}}}-3\right]^{1 / 2} \\
& =2.139 \sqrt{r_{\mathrm{s}} m e^{2} / \kappa} \\
\mathcal{S}_{(123) \rightarrow(312)} & =6 \sqrt{m \frac{e^{2}}{\kappa} r_{\mathrm{s}}} \int_{0}^{1 / 2} \mathrm{~d} q\left[\frac{1}{\sqrt{1-3 q+3 q^{2}}}-1\right]^{1 / 2} \\
& =2.852 \sqrt{r_{\mathrm{s}} m e^{2} / \kappa}
\end{aligned}
$$

are obtained assuming straight lines $\overrightarrow{\boldsymbol{x}}(q)$ for simplicity. This yields upper bounds to the true values of $\mathcal{S}$ which may be reasonable particularly for $\mathcal{S}_{(123) \rightarrow(312)}$. The estimate for $\mathcal{S}_{(123) \rightarrow(213)}$ is surely worse because the classical path $\overrightarrow{\boldsymbol{x}}(q)$ is more difficult to estimate for 
the pair exchange. The chosen paths for both cases are indicated in figure 2. Thus the pair exchange process (corresponding to the superexchange within the lattice description below) is slightly dominant compared to the ring process in the equilateral triangle. This supports a ground state of low total spin $S=1 / 2$. However, the triangle can easily be distorted so that $\mathcal{S}_{(123) \rightarrow(312)}$ is much reduced while $\mathcal{S}_{(123) \rightarrow(213)}$ remains almost unaffected, resulting in a crossover to a spin polarised ground state [31,32] (see also below).

The existence of ring exchange processes highlights a fundamental difference between a true lattice model and a continuum model. In order to accommodate such processes we need a better lattice approximation to the continuum than that suggested by the Wigner lattice. This may be achieved by introducing intermediate lattice points and corresponding localised one-electron orbitals. Orthogonalising these one-electron orbitals then enables an orthonormal many-electron basis set to be constructed, as before. This is shown in figure 3 for the triangular dot in which an extra lattice point is inserted between each pair of vertices.

The low-energy eigenstates may now be expanded in this basis set. As before, the probability of occupation of the three vertices is highest, leading to a $2^{3}=8$-fold degenerate ground-manifold when the hopping terms are switched off. Higher-lying base states, corresponding to double-occupation of a lattice site and to occupation of intermediate lattice sites may then be eliminated by degenerate perturbation theory, resulting once more in an effective spin-Hamiltonian. Intermediate states which involve double-occupation of a lattice site give rise to a Heisenberg superexchange term in the effective Hamiltonian, with $J=8 t^{4} / V^{2} U$, where $t, V$ and $U$ have the same meanings as before but now refer to nearestneighbor and on-site interactions for the new lattice. Longer-range interactions are ignored. (Note, however, that this is not necessary, it being straightforward to include longer-range interactions leading to a renormalisation of $J$ which will leave the form of the (Heisenberg) pair exchange unchanged.) There are two processes involved in this superexchange making equal contributions to $J$. These are shown in figure 4 . These processes are analogous to superexchange in atomic systems in which the intermediate sites would correspond to ligand ions surrounding transition-metal ions. Recent examples of this kind of system are the copper-oxide planes of the high-temperature superconductors, for which there is a similar fourth-order expression for the superexchange [33]. It is also clear from this new lattice description that other processes, which do not involve double occupation of a site (and therefore do not involve the exclusion principle), are potentially important. The lowest-order process of this type is also a pair exchange and occurs in fifth-order. An example is shown in figure 1 (b), which makes a contribution to $J$ of order $t^{5} / V^{4}$. Despite being higher-order than the other superexchange terms, this process is, in fact, the largest since it does not involve double-occupation of a site (i.e. there is no $U$-factor in the energy denominator).

With intermediate lattice points it is easy to see that there are ring processes giving rise to simultaneous rotation of all three electrons. One such process, in which the three electrons are rotated cyclically by $2 \pi / 3$, is shown in figure 5. The process resembles the classical path which would contribute to the WKB tunneling rate, cf. figure 2. The former is a sixth-order process giving rise to a cyclic rotation operator in the effective Hamiltonian with amplitude $t^{6} / 12 V^{5}$. Summing over all such processes results directly in an effective 'ring' operator, $-K\left(R_{2 \pi / 3}+R_{-2 \pi / 3}\right)$, where $K$ is a constant energy and $R_{2 \pi / 3}\left(R_{-2 \pi / 3}\right)$ is an operator which performs a cyclic (anticyclic) rotation of all three electrons (c.f. (3.32)). We note that these rotation operators may be written as spin-operators using the identities, 


$$
R_{2 \pi / 3} \equiv P_{12} P_{23} \text { and } R_{-2 \pi / 3} \equiv P_{23} P_{12}
$$

where $P_{i j} \equiv 2 \boldsymbol{s}_{1} \cdot \boldsymbol{s}_{2}+1 / 2$ is the Dirac exchange operator. Hence, the effective spinHamiltonian for 3 electrons in an equilateral triangular potential well becomes,

$$
H_{\mathrm{eff}}=\frac{J}{2}\left[\left(P_{12}-1\right)+\left(P_{23}-1\right)+\left(P_{31}-1\right)\right]-K\left(P_{12} P_{23}+P_{23} P_{12}\right) \quad .
$$

We see immediately from this form that the spin polarised state has eigenenergy $-2 K$. It is straightforward to complete the diagonalisation of $H_{\text {eff }}$ which results in a further pair of degenerate doublets at energy $K-3 J / 2$. Thus we see that the ring and exchange terms oppose each other with the former favouring a high-spin ground state and the latter a lowspin ground state. The crossover occurs at $J=2 K$, which is not quite reached for the equilateral triangle, as discussed above.

This will not always be so for other geometries as follows immediately from the fact that we can change the shape of the dot in such a way that the ring processes are increased in amplitude relative to the exchange processes. An 'extreme' example of this is the circular dot for which there is no barrier to the simultaneous rotation of the three electrons and the ground state should be spin polarised. The transition from high-spin to low-spin ground states in rings with increasing impurity barrier has been discussed recently [32 in the context of persistent currents.

With the inclusion of higher-order exchange processes involving also more than two particles, this completes the mapping of formerly continuous electron problems (2.1) onto lattice models (3.28) for their low energy and spin properties. This incorporates all permutational processes which according to the pocket-state description may be relevant. The above derivation of an effective-spin model for a triangular dot, including ring terms, is easily generalised to arbitrary shapes by inspecting all possible classes of ring and exchange terms on a suitably dense lattice into (3.28). The relative amplitudes of the various processes can be estimated semiclassically.

\section{COMPARISON WITH EXACT RESULTS AND OTHER APPROXIMATIONS}

To corroborate the lattice description for the low energy and spin properties of quantum dots we compare the results from (3.28) with results obtained by numerically exact diagonalisations [15] and by the pocket-state method [16].

The simplest model for an "artificial atom" is a hard wall box in one dimension. Rigorous properties for the sequence of spin states

$$
E(S)<E\left(S^{\prime}\right)
$$

for $S<S^{\prime}$ are known by a theorem of Lieb and Mattis 34]. $E(S)$ denotes the lowest

energy of $N$ almost arbitrarily interacting electrons to given spin $S=\left\{\begin{array}{c}0 \\ 1 / 2\end{array}\right\}, \ldots, N / 2$. In particular, the ground-state spin must be minimal. This rule (5.1) is in obvious agreement with the description in terms of an antiferromagnetic Heisenberg (spin-1/2) chain (3.30), valid for this case according to section [II], where the exchange of adjacent electrons is the 
relevant permutational process and there is only one classical minimum energy configuration, $(\nu=1)$. In table [ the energy values of $N=2,3,4$ electrons are given in units of $J$ as obtained within the pocket-state approximation (PSA), assuming $J_{12}=J_{23}=J_{34}$. These spectra agree exactly with the eigenvalues of the Heisenberg chain of $N=2,3,4$ spins apart from unimportant additive constants. Similarly, figure 6 shows the excitation energies for $N=3, \ldots, 6$ and includes also results obtained by numerical diagonalisation of the Hamiltonian matrices for $N=3,4$ (dashed). For the cases $N=5$ and $N=6$ we also checked, by numerical diagonalisation, that the Heisenberg chain shows exactly the same spectrum.

As a further example we investigated $N=2, \ldots, 5$ electrons in a two-dimensional square with hard walls. For these cases the interaction must be long range $w(|\boldsymbol{x}|) \sim|x|^{-\gamma}$ with $\gamma<2$ in order to make the PSA applicable. The spectra obtained within PSA are shown in figure 7. For $N=4$ the nearest neighbor pair exchange process has been compared with the competing process of all 4 electrons rotating cyclically by $\pi / 4$ [16], in a similar way as it has been done for $N=3$ electrons in a triangle in the previous section, with the result of the former being slightly dominant. In all cases the equilibrium positions of the electrons have been taken as sites for single electron states for the lattice description, as discussed in section [II], and the importance of transition processes has been estimated semiclassically. For $N=5$ one electron is, by electrostatics, located in the centre of the square being coupled antiferromagnetically, according to (3.30), to the 4 electrons placed in the corners. The Heisenberg model is appropriate since there is only one classical equilibrium configuration $(\nu=1)$. Neglecting next nearest neighbor exchange results in a $S=3 / 2$ ground state spin, using the PSA and permutation group theory. This result is immediately understandable from the Heisenberg spin-Hamiltonian, which clearly has a quartet ground state. Furthermore, we have checked that for all spectra, the corresponding Heisenberg $(N=4,5)$ or $t-J-V(N=2,3)$ Hamiltonians exactly reproduce the correct ordering of the spin multiplets and the correct energy splittings provided that the parameters $t, J$ and $V$ are chosen to be consistent with the corresponding tunnelling amplitudes in the PSA.

As a third example we look at the spectrum of three electrons in a one-dimensional ring. In section [V] we explained why the ground state is high spin $(S=3 / 2)$, which is also found in the pocket-state description. Two degenerate low spin $(S=1 / 2)$ excited states are higher in energy by the rotational constant $h^{2} / 2 m N L^{2}$, where $L$ is the circumference of the ring. They are additionally shifted by the magnitude of the pair exchange process $J$ towards lower energy, in agreement with (4.8).

\section{SUMMARY AND OUTLOOK}

Based on the pocket-state description of the low energy excitations in finite and a priori continuous systems of interacting electrons at low densities we have derived a lattice formulation for this problem. Non-orthogonal single electron states centred near the positions where the electrons would be in the classical minimum energy configurations are orthogonalised, leading to contributions from double occupancies $\sim U_{i} n_{i \uparrow} n_{i \downarrow}$ in an effective Hubbard model description. Subsequent elimination of high energy states $\left(\sim U_{i}\right.$ or $\sim V_{i j}$, from nearest neighbor occupations) yields effective antiferromagnetic Heisenberg (eq. (3.30) 
for $\nu=1$ ) or charge-spin Hamiltonians (eq. (3.28) for $\nu>1$ ) depending on the number $\nu$ of classical minimum energy configurations. An extended version of the Hubbard model must be considered if highly symmetric geometries or ring processes are important. A crucial enhancement has been to enable "ring exchange" processes to be included, corresponding to the cyclic permutation of $N>2$ electrons. Intermediate lattice points were introduced and the additional states corresponding to their single occupation were also eliminated by higher order perturbation theory, even though they are much lower in energy than states corresponding to double occupation. This yields generalized effective spin- $1 / 2$ models containing up to $(N-1)$-fold products of pairs of spin operators (see Eqn. 4.7). The magnitudes of the parameters for the superexchanges $J$ and the ring exchanges $K$ can be estimated semiclassically.

Apart from providing a quantitative description of the low-energy spectra in quantum dots the mapping onto lattice models is, in itself, very illuminating. For example, in cases that can be mapped onto the antiferromagnetic Heisenberg model for the low energy states, the spectrum consists of a level multiplet of 'band width' $N J$ where $J$, the nearest neighbor superexchange integral, depends exponentially on the mean electron distance $r_{\mathrm{s}}$. The highest energy state within this multiplet is the spin polarized state, in agreement with what has been derived within the pocket-state approximation using less obvious arguments. All spectra obtained by the lattice descriptions coincide exactly with those found in the pocket-state [16] approximation and are in very convincing agreement with results gained by numerical diagonalisations [15, 35]. The computational effort required for the former is, however, considerably less compared to both of the other methods. This will enable much larger systems with $N>20$ to be investigated.

In certain geometries of two dimensional quantum dots, $\nu>1$ classical configurations of lowest electrostatic energy may exist leading to an effective spin-charge model instead of a spin only effective model. Whilst such situations are straightforward to deal with by the lattice method, we should point out that in real experimental situations the high symmetry will be lowered by a polarizable environment for the following reason. The energetically most favourable locations for the quantum dot electrons depend on the distribution of surrounding (non-conducting) charges, which themselves are influenced by the distribution of the (Wigner localised but under transport conditions conducting) dot charges. Selfconsistency allows the dot electrons to lower the total energy by adjusting their environment. For example, two electrons in a square will easily break the two-fold symmetry of the classical ground-states by polarizing their surroundings, leaving a diamond shaped configuration for the potential. The interplay between the surroundings and the granular electron density of the dot finally tends to lower the number of classical low energy configurations. Only configurations with energies smaller than the band width $\sim N J$ eventually need to be considered explicitly. This plays a particular role e.g. in double-dot systems [36]. Additionally, one should keep in mind that the shape of the quantum dot also depends on voltages applied to side gates [37], so that the fine structure spectra may change with gate voltage.

The lattice description simplifies considerably the determination of excitations in quantum dots. These excited levels may be deduced implicitly from non-linear transport experiments [5]. Furthermore, transport is qualitatively influenced by the total spins of the many-electron states by two types of spin blockades [6, 38].

The occurrence of a non-minimal total spin for the ground state in a finite electron 
system is a direct consequence of the geometry of the boundary. For systems which can be represented by an antiferromagnetic Heisenberg model, this relationship is obvious (c.f. discussion of the square dot with $N=5$ in section $\nabla$ ). Apart from the electron number the spin values of ground and of excited states should depend very sensitively on the shape of the quantum dot. In future research this will be investigated systematically to allow specific suggestions for experimental design.

Another highly relevant topic for real experimental situations is to apply the mapping derived in the present work to study the interplay between mutual interactions of the quantum dot electrons and impurities. No reliable picture exists presently, which demonstrates our lack of understanding for the persistent currents observed in mesoscopic rings. Theory is still not capable of explaining even the order of magnitude of the high values found experimentally. It is known that one dimensional models of rings are insufficient to describe this problem [39]. Furthermore, evidence exists that the electron spin is important [40,32]. Our mapping onto lattice models demonstrates that calculations based on the Hubbard model 4143 are not significantly influenced by the lattice but indeed reproduce the low energy properties of the original continuous problems in presence of strong interactions correctly if the parameters, particularly the filling of the former, are interpreted accordingly. Advantage can be taken of the comparably large electron numbers that can be dealt with by our method, which would be otherwise intractable. This will allow the investigation of multiple-channels in two-dimensional rings of finite widths in the presence of impurities and flux.

\section{Acknowledgements}

We acknowledge stimulating discussions with Bernhard Kramer, Colin Lambert, Walter

Stephan and colleagues in our EU-sponsored HCM network on the quantum dynamics of phase coherent structures. (HCM No. CHRX-CT93-0136) 


\section{REFERENCES}

[1] D. V. Averin, K. K. Likharev, J. Low Temp. Phys. 62, 345 (1986), H. Grabert, M. Devoret, editors "Single Charge Tunneling", NATO ASI Series, Plenum Press, volume 294 (1992) and references therein, Special Issue on Single Charge Tunneling ed. by H. Grabert, Z. Phys. B 85, 317-468 (1991).

[2] Ch. Sikorski, U. Merkt, Phys. Rev. Lett. 62, 2164 (1989).

[3] B. Meurer, D. Heitmann, K. Ploog, Phys. Rev. Lett. 68, 1371 (1992), D. Heitmann, J. P. Kotthaus, in Physics Today, Vol. 56, June (1993).

[4] U. Meirav, M. A. Kastner, S. J. Wind, Phys. Rev. Lett. 65, 771 (1990).

[5] D. V. Averin, A. N. Korotkov, J. Low Temp. Phys. 80, 173 (1990), A. T. Johnson, L. P. Kouwenhoven, W. de Jong, N. C. van der Vaart, C. J. P. M. Harmanns, C. T. Foxon, Phys. Rev. Lett. 69, 1592 (1992),

J. Weis, R. J. Haug, K. v. Klitzing, K. Ploog, Phys. Rev. Lett. 71, 4019 (1993),

E. B. Foxman, P. L. McEuen, U. Meirav, N. S. Wingreen, Y. Meir, P. A. Belk, N. R. Belk, M. A. Kastner, S. J. Wind, Phys. Rev. B 47, 10020 (1993).

[6] D. Weinmann, W. Häusler, W. Pfaff, B. Kramer, U. Weiss, Europhys. Lett. 26, 689 (1994),

W. Pfaff, D. Weinmann, W. Häusler, B. Kramer, U. Weiss, Z. Phys. B 96, 201 (1994).

[7] C. Bruder, H. Schoeller, Phys. Rev. Lett. 72, 1076 (1994),

L. P. Kouwenhoven, S. Jauhar, K. McCormick, D. Dixon, P. L. McEuen, Yu. V. Nazarov, N. C. van der Vaart, C. T. Foxon,, Phys. Rev. B 50, 2019 (1994).

[8] U. Merkt p. 165 in "The Physics of Few-Electron Nanostructures", ed. by L. J. Geerligs, C. J. P. M. Harmans, L. P. Kouwenhoven, North Holland, Physica B, Vol. 189 (1993).

[9] A. V. Chaplik, JETP Lett. 52, 31 (1990).

[10] M. Wagner A. V. Chaplik and U. Merkt, Phys. Rev. B 51, 13817 (1995).

[11] M. A. Kastner, Rev. Mod. Phys. 64, 849 (1992).

[12] D. Pfannkuche, V. Gudmundsson, P. A. Maksym, Phys. Rev. B 47, 2244 (1993).

[13] P. A. Maksym, T. Chakraborty, Phys. Rev. Lett. 65, 108 (1990),

U. Merkt, J. Huser, M. Wagner, Phys. Rev. B 43, 7320 (1991),

D. Pfannkuche, R. R. Gerhards, Phys. Rev. B 44, 13132 (1991),

W. Häusler, B. Kramer, J. Mašek, Z. Phys. B 85, 435 (1991),

P. Hawrylak, D. Pfannkuche, Phys. Rev. Lett. 70, 485 (1993).

[14] P. A. Maksym Physica B 184, 385 (1993),

P. A. Maksym Europhys. Lett. 31, 405 (1995),

P. A. Maksym Phys. Rev. B (In Press).

[15] W. Häusler, B. Kramer, Phys. Rev. B 47, 16353 (1993).

[16] W. Häusler, Advances in Solid State Physics 34, 171 (1994),

W. Häusler, to appear in Z. Phys. B (1995).

[17] K. A. Chao, J Spalek, A. M. Oles, Phys. Rev. B 18, 3453 (1978).

[18] J. H. Jefferson, J. Phys. C 21, 1193 (1988).

[19] D. E. Rutherford, "Substitutional Analysis",

The Edinburgh University Press, London (1948), 
M. Hamermesh, "Group Theory and its Applications to Physical Problems", Addison-Wesley, Massachusetts (1962); new edition by Dover Publications, New York, (1989).

[20] J. Hubbard, Proc. Royal Soc. London A 276, 238 (1963).

[21] E. P. Wigner, Phys. Rev. 46, 1002 (1934).

[22] E. Dagotto, Rev. Mod. Phys. 66, 763 (1994).

[23] K. Jauregui, W. Häusler, B. Kramer, Europhys. Lett. 24, 581 (1993).

[24] A. Hüller, Z. Phys. B 36, 215 (1980).

[25] P. O. Löwdin, J. Chem. Phys. 18, 365 (1950).

[26] I. Lindgren, J. Phys. B: At. Mol. Phys. 7, 2441 (1974).

[27] J. R. Schrieffer, P. A. Wolff, Phys. Rev. 149, 491 (1966).

[28] It is possible to include the $V$-term in the unperturbed Hamiltonian. However, this introduces complications in the perturbation expansions (since the intermediate state energies depend on their environment) which are best avoided. Since we are interested in the regime for which $U \gg V$, including $V$ in the perturbation introduces only a small error.

[29] L. F. Feiner, J. H. Jefferson, and R. Raimondi, Phys. Rev. B 51, 12797 (1995).

[30] F. C. Zhang and T. M. Rice Phys. Rev. B 37, 3757 (1988),

J. H. Jefferson, Physica B 165-166, 1013 (1990),

J. H. Jefferson, H. Eskes, and L. F. Feiner, Phys. Rev. B 45, 7959 (1992),

H.-B. Schüttler and A. J. Fedro, Phys. Rev. B 45, 7588 (1992),

C. D. Batista and A. A. Aligia, Phys. Rev. B 47, 8929 (1993),

V. I. Belinicher, A. L. Chernyshev, and L. V. Popovich, Phys. Rev. B 50, 13768 (1994),

L. F. Feiner, J. H. Jefferson, and R. Raimondi, Phys. Rev. B (In Press),

R. Raimondi, J. H. Jefferson and L. F. Feiner, Phys. Rev. B (In Press).

[31] K. W. H. Stevens, J. Phys. C 16, 5765 (1983).

[32] W. Häusler, B. Kramer p. 169 in 'Quantum Dynamics of Submicron Structures', ed. by H. A. Cerdeira, B. Kramer, G. Schön, NATO ASI Series E, Applied Sciences, Vol. 291, Kluwer, Dordrecht (1995),

W. Häusler, Physica B (In Press).

[33] H. Eskes and J. H. Jefferson, Phys. Rev. B 48, 9788 (1993).

[34] E. Lieb, D. Mattis, Physical Review 125, 164 (1962).

[35] G. W. Bryant, Phys. Rev. Lett. 59, 1140 (1987).

[36] N. C. van der Vaart, S. F. Godijn, Yu. V. Nazarov, C. J. P. M. Harmans, J. E. Mooij, L. W. Molenkamp and C. T. Foxon, Phys. Rev. Lett. 74, 4702 (1995),

F. Hofmann, T. Heinzel, D. A. Wharam, J. P. Kotthaus, G. Böhm, W. Klein, G. Tränkle, G. Weimann, Phys. Rev. B 51, 13872 (1995),

R. H. Blick, R. J. Haug, J. Weis, D. Pfannkuche, K. v. Klitzing and K. Eberl, preprint (1995).

[37] L. P. Kouwenhoven, PhD thesis, Technical University of Delft (1992).

[38] D. Weinmann, W. Häusler, B. Kramer, Phys. Rev. Lett. 74, 984 (1995).

[39] R. Berkovits, Y. Avishai, Europhys. Lett. 29, 475 (1995).

[40] T. Giamarchi, B. S. Shastry, Phys. Rev. B 52, 14809 (1995).

[41] N. Yu and M. Fowler, Phys. Rev. B 45, 11795 (1992).

[42] M. Ramin, B. Reulet, H. Bouchiat, Phys. Rev. B 51, 5582 (1995). 
[43] R. Römer, A. Punnoose, Phys. Rev. B 52, 14809 (1995). 


\section{TABLES}

\begin{tabular}{cccc}
\hline \hline$N$ & $S$ & $E_{m}^{(N)}-E_{\text {Ground state }}^{(N)}$ \\
\hline 2 & 0 & 0 \\
2 & 1 & $J^{(2)}$ \\
3 & $1 / 2$ & 0 \\
3 & $1 / 2$ & $J^{(3)}$ \\
3 & $3 / 2$ & $(3 / 2) J^{(3)}$ \\
4 & 0 & 0 \\
4 & 1 & $(1+\sqrt{3}-\sqrt{2}) J^{(4)} / 2$ \\
4 & 1 & $(1+\sqrt{3}) J^{(4)} / 2$ \\
4 & 0 & $(2 \sqrt{3}) J^{(4)} / 2$ \\
4 & 1 & $(1+\sqrt{3}+\sqrt{2}) J^{(4)} / 2$ \\
4 & 2 & $(3+\sqrt{3}) J^{(4)} / 2$ \\
\hline \hline
\end{tabular}

TABLE I. Analytical values for the fine structure spectrum $E_{m}^{(N)}$ of $N$ interacting electrons in a one-dimensional hard wall box within PSA for $N \leq 4 . S$ is the total spin of $N$ Fermions with $s=1 / 2$. The excitation energies $E_{m}^{(N)}-E_{\text {Ground state }}^{(N)}$, are given in units of $J^{(N)}$. 


\section{FIGURES}

FIG. 1. Classical low-energy configurations for two electrons in a 2D square quantum well. The first two configurations are lowest in energy whilst the remaining four are $V$ higher in energy due to Coulomb repulsion. All other configurations have higher electrostatic energy.

FIG. 2. (a) Classical ground-state configuration for three electrons in a triangular quantum dot. (b) Classical path for the exchange of two electrons. (c) Classical path for the cyclic permutation of all three electrons. Particles are shown in intermediate positions along their trajectories.

FIG. 3. Lattice points for the triangular quantum dot. In the ground manifold all three electrons will be close to the positions indicated by the circles. Excited (intermediate) states correspond to one or more electrons in localised orbitals centered on the crosses.

FIG. 4. Superexchange processes. (a) Fourth-order $\left(\sim t^{4} / V^{2} U\right)$. (b) Fifth-order $\left(\sim t^{5} / V^{4}\right)$.

FIG. 5. A six-step ring process for three electrons in a triangular quantum dot. The electrons would move continuously and simultaneously in the direction of the arrows in the WKB approximation.

FIG. 6. Fine structure multiplets of a quasi one-dimensional quantum dot, for $N=3, \ldots, 6$ as obtained directly from the pocket-state approximation and in exact agreement with the eigensolutions of the effective spin Hamiltonian. The dashed lines were obtained by direct numerical diagonalisation of the interacting electron problem. The $N$-dependence of $J^{(N)}$ is not considered and $J$ has been adjusted to normalize the 'bandwidth' of the multiplets.

FIG. 7. Fine structure spectra of (a) $N=3$, (b) $N=4$ and (c) $N=5$ electrons in a 2D square as obtained within the pocket-state approximation. In (a) the dominant tunneling process $(t)$ corresponds to nearest-neighbor hopping of an electron whereas in (b) and (c) the dominant tunneling process is nearest-neighbor exchange $(J)$. All spectra are identical with those obtained from the corresponding charge-spin Hamiltonian. 